\title{
INFLUÊNCIA DA CIANAMIDA HIDROGENADA NA BROTAÇÃO E PRODUÇÃO DA VIDEIRA 'NIAGARA ROSADA' NA REGIÃO OESTE DO PARANÁ1
}

\author{
TATIANA WERLE ${ }^{2}$, VANDEIR FRANCISCO GUIMARÃES ${ }^{3}$, IDIANA MARINA DALASTRA², \\ MÁRCIADE MORAES ECHER ${ }^{3}$, RAFAEL PIO ${ }^{3}$
}

RESUMO - As videiras apresentam um período de dormência que requer certa quantidade de frio para retomar seu desenvolvimento na primavera. Apesar da eficiência da cianamida hidrogenada na indução e uniformização de brotações na videira, ainda faltam estudos específicos para determinar a melhor dosagem, pois esta varia de acordo com a condição climática e a época de aplicação. Dessa forma, justifica-se este estudo para a região oeste do Paraná. O experimento foi conduzido no município de Quatro Pontes - PR, sendo utilizadas 6 doses de cianamida hidrogenada $\left(\mathrm{H}_{2} \mathrm{CN}_{2}\right): 0 ; 10 ; 20 ; 30 ; 40$ e $50 \mathrm{~mL} \mathrm{~L}^{-1}$. A aplicação foi realizada no dia 16 de agosto de 2005, por meio de pincelamento de gemas. A aplicação de cianamida hidrogenada proporcionou maior uniformização da emissão de brotações da 'Niagara Rosada', antecipando em aproximadamente 14 dias a brotação das gemas nos tratamentos em relação à testemunha. A maior porcentagem de brotação foi obtida com o tratamento em que se aplicou a concentração de $20 \mathrm{~mL} \mathrm{~L}^{-1}$ do produto. Doses crescentes de cianamida hidrogenada não causaram efeito na produtividade e qualidade da fruta.

Termos para Indexação: Vitis labrusca L., dormência e bioativador.

\section{INFLUENCE OF HYDROGENOUS CYANAMIDE IN THE SPROUTING AND PRODUCTION OF 'NIAGARA ROSADA' GRAPEVINE, IN THE WEST REGION OF THE STATE OF PARANÁ}

ABSTRACT - The vines present a dormant period which requires a chilling time to retake their development in the spring. Despite the hydrogen cyanamide efficiency in the vine sprouting, specific studies to determine the best dosage are still lacking, for it varies according to the region and application time. So, this study is justified in the west region of the State of Paraná. This experiment established six treatments of hydrogen cyanamide dosages $\left(\mathrm{H}_{2} \mathrm{CN}_{2}\right): 0,10,20,30,40$ and $50 \mathrm{~mL} \mathrm{~L}^{-1}$. The application was performed in August 16, 2005, by brushing the dormant buds. The application of hydrogen cyanamide provided a larger sprouting of the 'Niagara Rosada', providing an advance of nearly 14 days in relation to the control.The largest sprouting percentage was obtained with the treatment of $2 \mathrm{~mL} \mathrm{~L}^{-1}$. For the variables concerning to quality and productivity, there were no statistic differences among the treatments. Index Terms: Vitis vinifera L., dormancy, bioactivator.

\section{INTRODUÇÃO}

As frutíferas de clima temperado, a priori, as viníferas, apresentam um período de dormência que requer certa quantidade de horas de frio para retomar seu desenvolvimento na primavera. Uma vez a endodormência das gemas tenha sido estabelecida, a brotação e a floração das gemas serão uniformes (Pires, 1998). Em regiões onde o frio hibernal não é suficiente para superar a endodormência das gemas, como é o caso do oeste do Paraná, torna-se necessária a utilização de compostos químicos para o suprimento artificial das unidades de frio impostas pelas gemas, garantindo brotação abundante e uniforme das gemas (Petri et al., 1996).

A cianamida hidrogenada $\left(\mathrm{H}_{2} \mathrm{CN}_{2}\right)$ pode ser utilizada para o suprimento artificial da endodormência das gemas (Shulman et al., 1986; George \& Nissen, 1993). Sabe-se que a cianamida hidrogenada é rapidamente absorvida e metabolizada e que sua ação causa diminuição da atividade da catalase, sem modificar a peroxidase, o que resulta em um aumento da concentração de peróxido $\left(\mathrm{H}_{2} \mathrm{O}_{2}\right)$ nas gemas (Shulman et al., 1986; Goldback et al., 1988). Esse aumento é responsável pela ativação do ciclo das pentoses e conseqüente indução da saída da dormência das gemas (Omran, 1980).

O emprego de agroquímicos é indispensável para a melhoria da produtividade e qualidade da produção em viticultura tropical. Especial destaque deve ser dado aos tratamentos à base de cianamida que, através do estímulo à quebra da dormência das gemas, permitiu um grande avanço da viticultura nas regiões tropicais. Utilizados nas dosagens e épocas adequadas, os reguladores vegetais atuam diretamente no desenvolvimento vegetativo e reprodutivo das plantas (Pires, 1998).

Na região de Jundiaí - SP, Pires et al. (1999) constataram que a pulverização das gemas da videira 'Niagara Rosada', com cianamida hidrogenada, adiantou a brotação das gemas e aumentou a porcentagem de gemas brotadas, o número de cachos e a produtividade por planta. No Rio Grande do Sul, Miele (1991) obteve resultados semelhantes em videiras 'Cabernet

\footnotetext{
(Trabalho 096-07).Recebido em: 10-04-2007. Aceito para publicação em: 30-11-2007.

${ }^{2}$ Eng. Agr., Mestrando em Produção Vegetal, Universidade Estadual do Oeste do Paraná (UNIOESTE). Rua Pernambuco, nº 1777, C.P. 1008, Centro, 85960-000, Marechal Cândido Rondon-PR. tatiwerle@yahoo.com.brididalastra@yahoo.com.br

${ }^{3}$ Eng $^{\circ}$. Agrônomo, D.Sc., Professor Adjunto da Universidade Estadual do Oeste do Paraná (UNIOESTE). Rua Pernambuco, n ${ }^{\circ}$ 1777, C.P. 1008, Centro, 85960-000, Marechal Cândido Rondon-PR.Bolsista de Produtividade em Pesquisa do CNPq. Autor para correspondência: :rafaelpio@hotmail.com.,vandeirfg@yahoo.com.br,mmecher@bol.com.br.
} 
Sauvignon'. Reddy \& Shikhamany (1989) pesquisaram o efeito da cianamida hidrogenada na quebra de dormência e brotação da videira 'Thompson Seedless' em condições tropicais, sendo que verificaram que o tratamento com $\mathrm{H}_{2} \mathrm{CN}_{2}$ a $3 \%$ resultou em alta porcentagem de brotação, com incremento de $63 \% \mathrm{em}$ comparação à testemunha.

Neste contexto, o presente trabalho teve como objetivos verificar a dose ideal da cianamida hidrogenada na indução e uniformização da emissão de brotações, qualidade e produtividade da videira 'Niagara Rosada', nas condições da região oeste do Paraná.

\section{MATERIAL E MÉTODOS}

O experimento foi conduzido em vinhedo comercial, localizado no município de Quatro Pontes, região oeste do Estado do Paraná. O local situa-se a aproximadamente $472 \mathrm{~m}$ de altitude nos paralelos de $24^{\circ} 35^{\prime} 42^{\prime \prime}$ latitude sul e $53^{\circ} 59^{\prime} 54^{\prime \prime}$ longitude oeste.

A videira 'Niagara Rosada' foi conduzida em sistema de latada e espaçamento de $1,5 \mathrm{~m} \times 3 \mathrm{~m}$, sendo que se encontravam no $2^{\circ}$ ano de produção, enxertadas sobre o porta-enxerto IAC 420-A.

O experimento foi implantado em delineamento de blocos ao acaso, com seis tratamentos, compostos pelas seguintes doses de cianamida hidrogenada $\left(\mathrm{H}_{2} \mathrm{CN}_{2}\right): 0 ; 10 ; 20 ; 30 ; 40$ e 50 $\mathrm{mL} \mathrm{L}^{-1}$, utilizando o produto comercial Dormex ${ }^{\circledR}$. Foram utilizadas quatro repetições por tratamento, sendo cada planta uma parcela experimental. A aplicação da cianamida hidrogenada foi realizada no dia 16 de agosto de 2005, dois dias após a poda curta. Foi aplicada diretamente às gemas, através de pincelamento.

Foram realizadas as seguintes avaliações: data de brotação, porcentagem de gemas brotadas, número médio de brotações por planta, diâmetro e comprimento médio da brotação. Por ocasião da colheita (aproximadamente $14^{\circ}$ Brix), foram escolhidos dois cachos ao acaso de cada planta, para a determinação das seguintes variáveis: massa média do cacho (g), comprimento do cacho $(\mathrm{cm})$, número de bagas por cacho, massa de bagas por cacho $(\mathrm{g})$, diâmetro médio da baga $(\mathrm{cm})$, massa da ráquis $(\mathrm{g})$, comprimento da ráquis $(\mathrm{cm})$, largura do ombro, teor de sólidos solúveis ( ${ }^{\circ}$ Brix), massa de cacho por planta e produtividade ( $\mathrm{kg} /$ planta).

\section{RESULTADOS E DISCUSSÃO}

A ausência da aplicação com a cianamida hidrogenada acarretou atraso para o início das brotações por planta, em relação aos demais tratamentos; aos 14 DAA (dias após a aplicação), todos os tratamentos com cianamida hidrogenada já apresentavam gemas brotadas. A utilização de $50 \mathrm{~mL} \mathrm{~L}^{-1}$ de cianamida hidrogenada favoreceu a antecipação no desenvolvimento inicial dos brotos. Aos 44 DAA, houve um aumento do número de brotações nos tratamentos em que foram aplicadas as concentrações de $20 \mathrm{~mL} \mathrm{~L}^{-1}$ e $40 \mathrm{~mL} \mathrm{~L}^{-1}$, quando comparadas aos 38 DAA (Figura 1).

$\mathrm{O}$ adiantamento na emissão de brotações das plantas que receberam o tratamento com cianamida hidrogenada em relação à ausência do bioativador, foi de aproximadamente 14 dias, ponto em que foram constatadas $50 \%$ de gemas brotadas. Esse adiantamento pode ocasionar antecipação da safra, mas, por outro lado, pode trazer sérias implicações quanto à ocorrência de baixas temperaturas e geadas no período em que as gemas se encontram brotadas, podendo ocasionar a morte das mesmas.

Para a porcentagem de gemas brotadas, pôde-se observar que a testemunha apresentou valores mais baixos $(64,6 \%)$ quando comparada às plantas tratadas com cianamida hidrogenada, que apresentaram em média $89,8 \%$ das gemas brotadas (Figura 2). $\mathrm{O}$ tratamento que obteve maior porcentagem de gemas brotadas, foi o que recebeu aplicação de $20 \mathrm{~mL} \mathrm{~L}^{-1}$, resultando em $93,6 \%$ das gemas brotadas.

Os resultados obtidos neste trabalho concordam com os obtidos por Pires et al. (1999), na região de Jundiaí - SP, que constataram que a pulverização das gemas da videira 'Niagara Rosada' com cianamida hidrogenada adiantou a brotação das gemas e aumentou a porcentagem de gemas brotadas. Resultados semelhantes foram obtidos por Miele (1991), no Rio Grande do Sul, com videiras 'Cabernet Sauvignon', sendo que as melhores doses de cianamida hidrogenada, para as variáveis estudadas, se situaram entre 1,8 e $1,9 \%$.

Houve diferença significativa entre os tratamentos no que diz respeito ao número de brotações (Figura 3). Nas plantas em que não foi aplicada a cianamida hidrogenada (testemunha), ocorreu $64 \%$ de gemas brotadas ( 24 gemas brotadas, de um total de 37). Já nas plantas onde foram aplicadas doses de cianamida hidrogenada, houve, em média, $89 \%$ de gemas brotadas ( 35 gemas brotadas de um total de 39). Segundo Pires (1998), a utilização de biorreguladores, nas dosagens e épocas adequadas, atua diretamente no desenvolvimento vegetativo e reprodutivo das plantas, confirmando os resultados deste experimento.

Observa-se diferença do número médio de brotações em função das doses de cianamida hidrogenada, aos 35 DAA e aos 38 DAA (Figura 4). Melhores resultados foram obtidos com a dose de $30 \mathrm{~mL} \mathrm{~L}^{-1}$, aos 35 DAA (Figura 4a) e aos 38 DAA (Figura 4b). Nota-se que, na ausência da aplicação do biorregulador, o número de brotações foi inferior em relação aos demais tratamentos. Nesse mesmo período, foi constatado menor número médio das brotações com a utilização de $50 \mathrm{~mL} \mathrm{~L}^{-1}$ de cianamida hidrogenada.

Houve aumento do diâmetro da brotação em relação ao aumento das dosagens de cianamida hidrogenada (Figura 5). Com $50 \mathrm{~mL} \mathrm{~L}^{-1}$ do biorregulador, além da redução no número de brotações observado na Figura 4, a dosagem em questão ocasionou redução no diâmetro das brotações (Figura 5). Em relação à testemunha, o menor diâmetro deve-se ao fato de que houve atraso na brotação desta em relação aos demais tratamentos, ocasionando, assim, diâmetro inferior em relação aos tratamentos com cianamida hidrogenada

Para o comprimento das brotações, a testemunha apresentou o menor comprimento (Figura 6). O maior comprimento foi observado quando foi utilizada a dose de $30 \mathrm{~mL} \mathrm{~L}^{-1}$. Tanto o diâmetro quanto o comprimento das brotações da videira dão uma idéia do vigor dos ramos, podendo estes terem relação com 
a qualidade e produtividade das plantas.

Embora as aplicações de cianamida hidrogenada tivessem apresentado um efeito na brotação das gemas dormentes, verificou-se que não houve diferenças significativas para as variáveis relacionadas aos frutos (Tabelas 1 e 2).

Esses resultados concordam com Botelho et al. (2002), que verificaram que as aplicações de cianamida hidrogenada tiveram um efeito significativo na brotação das gemas dormentes, mas não se verificou diferença significativa na produção total, possivelmente porque as varas não-tratadas, apesar de apresentarem uma porcentagem de brotação menor, ainda produziram ramos em número e nível de desenvolvimento adequados para garantir uma produção satisfatória, em função das condições climáticas mais apropriadas.

TABELA 1 - Número de bagas por cacho (NB), massa da ráquis (MR), massa de baga por cacho (MB), massa do cacho (MC), massa de cachos por planta (MCP) e produtividade (P) de uva 'Niagara Rosada', em função da aplicação de doses de cianamida hidrogenada. UNIOESTE, Quatro Pontes - PR, 2007.

\begin{tabular}{lllllll}
\hline $\begin{array}{l}\text { Doses de cianamida } \\
\text { hidrogenada }\end{array}$ & $\mathrm{NB}^{*}$ & $\mathrm{MR}(\mathrm{g})$ & $\mathrm{MB}(\mathrm{g})$ & $\mathrm{MC}(\mathrm{g})$ & $\mathrm{MCP}(\mathrm{kg})$ & $\mathrm{P}\left(\mathrm{t} \mathrm{ha}^{-1}\right)$ \\
\hline $0\left(\mathrm{~mL} \mathrm{~L}^{-1}\right)$ & 36,6 & 4,4 & 149,6 & 154,1 & 4,67 & 10,38 \\
$10\left(\mathrm{~mL} \mathrm{~L}^{-1}\right)$ & 43,1 & 4,3 & 153,4 & 157,7 & 5,42 & 12,05 \\
$20\left(\mathrm{~mL} \mathrm{~L}^{-1}\right)$ & 47,5 & 5,5 & 198,1 & 203,6 & 5,04 & 11,19 \\
$30\left(\mathrm{~mL} \mathrm{~L}^{-1}\right)$ & 42,1 & 4,7 & 170,6 & 175,3 & 5,70 & 12,66 \\
$40\left(\mathrm{~mL} \mathrm{~L}^{-1}\right)$ & 47,1 & 5,2 & 189,4 & 194,6 & 4,68 & 10,40 \\
$50\left(\mathrm{~mL} \mathrm{~L}^{-1}\right)$ & 44,9 & 5,1 & 177,0 & 182,1 & 4,60 & 10,21 \\
\hline $\mathrm{CV}(\%)$ & 16,9 & 20,8 & 22,7 & 22,6 & 28,3 & 28,3 \\
\hline
\end{tabular}

* Não foi observada diferença estatística pelo teste de Tukey, ao nível de $5 \%$ de probabilidade.

TABELA 2- Teor de sólidos solúveis totais (SST), diâmetro da baga (DB), comprimento da ráquis (CR), largura do ombro (LO) e comprimento do cacho (CC) por planta de uva 'Niagara Rosada', em função da aplicação de doses de cianamida hidrogenada. UNIOESTE, Quatro Pontes - PR, 2007

\begin{tabular}{llllll}
\hline $\begin{array}{c}\text { Doses de cianamida } \\
\text { hidrogenada }\end{array}$ & SST (\%)* & DB $(\mathrm{mm})$ & $\mathrm{CR}(\mathrm{cm})$ & LO $(\mathrm{cm})$ & $\mathrm{CC}(\mathrm{cm})$ \\
\hline $0\left(\mathrm{~mL} \mathrm{~L}^{-1}\right)$ & 15,8 & 17,2 & 8,3 & 6,4 & 10,3 \\
$10\left(\mathrm{~mL} \mathrm{~L}^{-1}\right)$ & 16,2 & 17,0 & 7,9 & 7,3 & 11,0 \\
$20\left(\mathrm{~mL} \mathrm{~L}^{-1}\right)$ & 16,2 & 17,4 & 8,4 & 7,9 & 11,1 \\
$30\left(\mathrm{~mL} \mathrm{~L}^{-1}\right)$ & 16,0 & 17,0 & 8,0 & 6,9 & 10,5 \\
$40\left(\mathrm{~mL} \mathrm{~L}^{-1}\right)$ & 16,1 & 17,0 & 8,5 & 6,7 & 11,3 \\
$50\left(\mathrm{~mL} \mathrm{~L}^{-1}\right)$ & 15,9 & 17,4 & 8,2 & 6,9 & 10,8 \\
\hline $\mathrm{CV}(\%)$ & 4,5 & 4,5 & 9,8 & 11,4 & 10,0 \\
\hline
\end{tabular}

* Não foi observada diferença estatística pelo teste de Tukey, ao nível de $5 \%$ de probabilidade.
Quanto ao teor de sólidos solúveis totais (SST), notouse que, em todos os tratamentos, o mesmo apresentava-se acima do recomendado para a colheita ( $14^{\circ}$ Brix). Pode-se observar que, na testemunha, apesar do atraso para o início da brotação das gemas em relação às plantas que receberam doses de cianamida hidrogenada, a maturação ocorreu de maneira semelhante aos demais tratamentos.

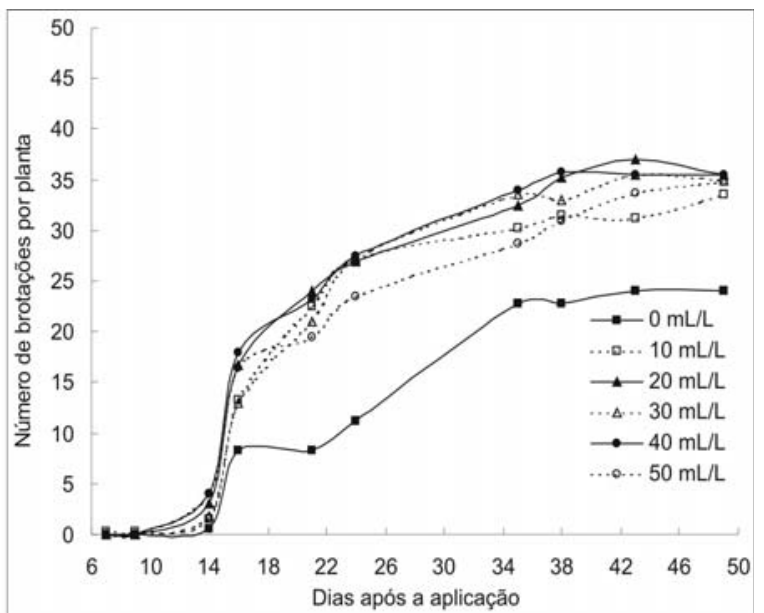

FIGURA 1- Número de brotações por plantas de videira 'Niagara Rosada' em função do tempo e em resposta após a aplicação de doses de cianamida hidrogenada. UNIOESTE, Quatro Pontes - PR, 2007.

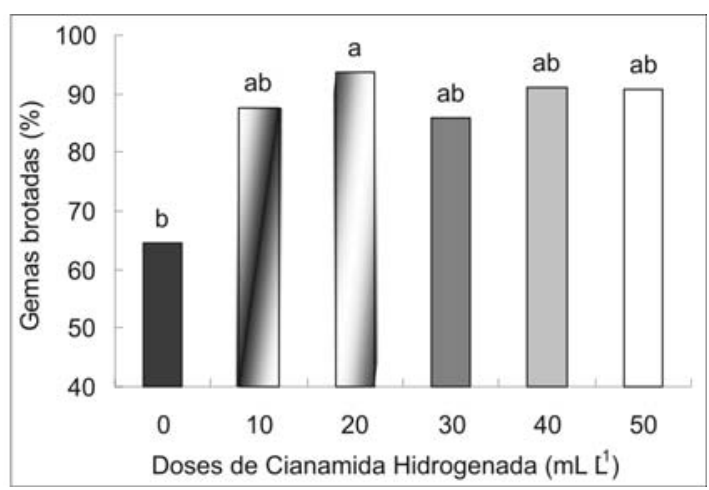

FIGURA 2 - Porcentagem final de gemas brotadas de videira 'Niagara Rosada' em resposta à aplicação de doses de cianamida hidrogenada. UNIOESTE, Quatro Pontes - PR, 2007. * cv $(\%)=18,71$. 


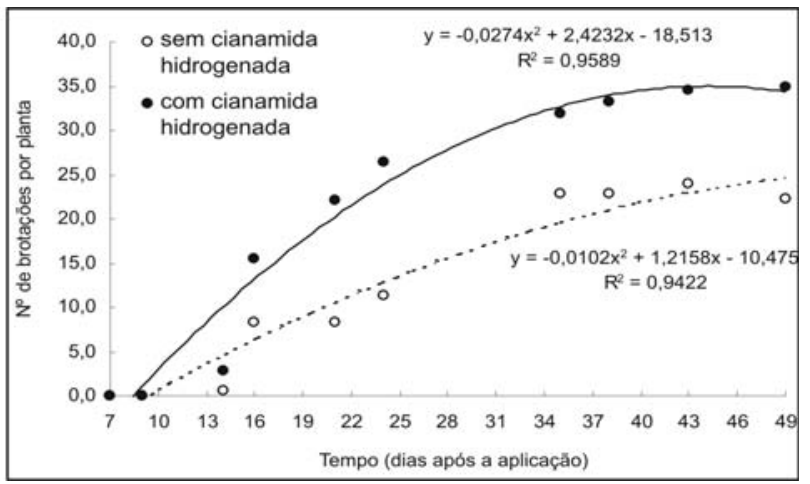

FIGURA 3 - Número de brotações por planta de videira 'Niagara Rosada' em função do tempo, com e sem a aplicação de doses de cianamida hidrogenada. UNIOESTE, Quatro Pontes - PR, 2007.
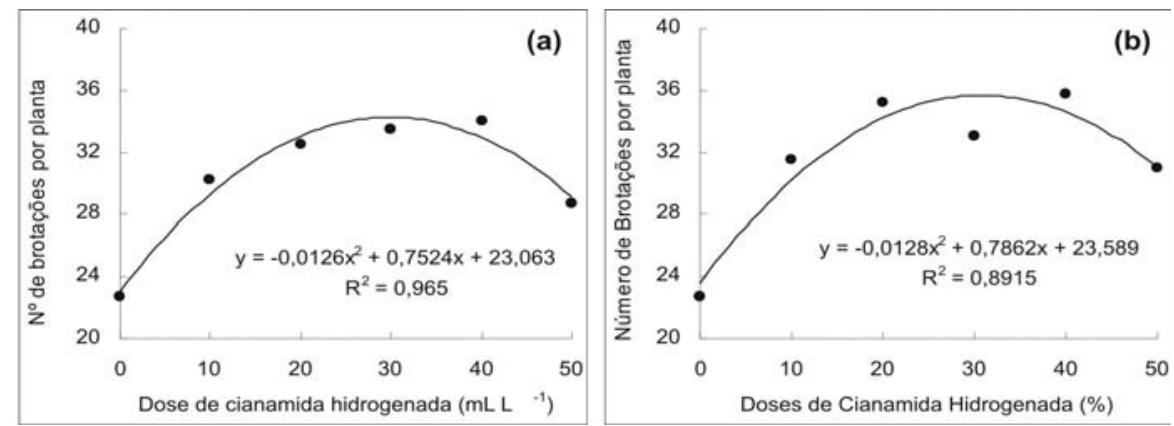

FIGURA 4 - Número de brotações em plantas de videira 'Niagara Rosada' em resposta à aplicação de doses de cianamida hidrogenada.

(a) 35 dias após a aplicação e (b) - 38 dias após a aplicação. UNIOESTE, Quatro Pontes - PR, 2007.

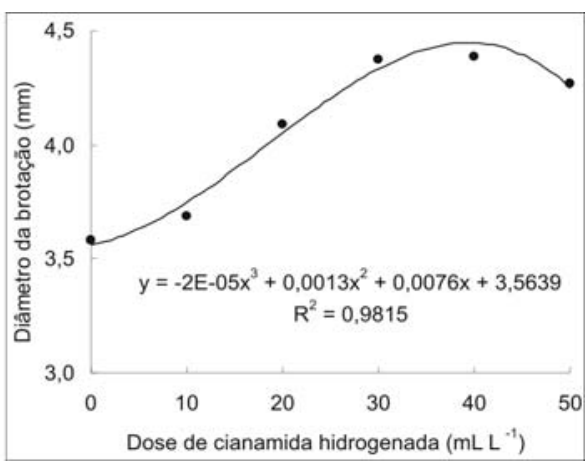

FIGURA 5 - Diâmetro de brotações de plantas de videira 'Niagara Rosada' em resposta à aplicação de doses de cianamida hidrogenada, aos 38 dias após a aplicação. UNIOESTE, Quatro Pontes - PR, 2007.

\section{CONCLUSÕES}

1- A aplicação de cianamida hidrogenada proporcionou maior uniformização da emissão de brotações da videira 'Niagara Rosada', nas condições climáticas do oeste do Paraná.

2 - Houve adiantamento de aproximadamente 14 dias na emissão de brotações das gemas.

3- A maior porcentagem de brotação foi obtida com o tratamento em que se aplicou a concentração de $20 \mathrm{~mL} \mathrm{~L}^{-1}$ de cianamida hidrogenada.

4- A aplicação de cianamida hidrogenada, através de pincelamento das gemas, não resultou em efeito sobre a qualidade e produtividade da videira 'Niagara Rosada'.

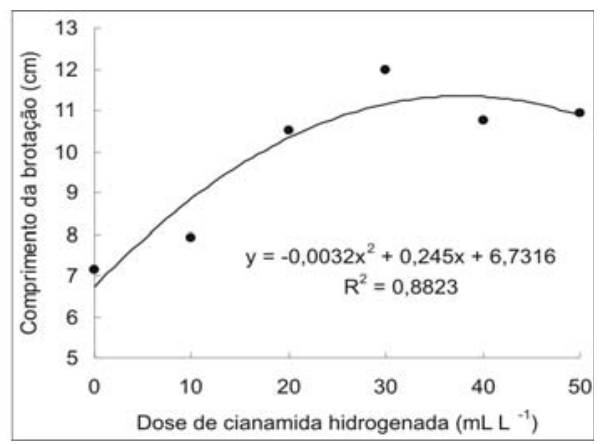

FIGURA 6 - Comprimento de brotações de plantas de videira 'Niagara Rosada' em resposta à aplicação de doses de cianamida hidrogenada, aos 38 dias após a aplicação. UNIOESTE, Quatro Pontes - PR, 2007.

\section{REFERÊNCIAS}

BOTELHO, R.V.; PIRES, E.J.P.; TERRA, M.M. Brotação e produtividade de videiras da cultivar Centenial Seedless (Vitis vinifera L.) tratadas com cianamida hidrogenada na região noroeste do Estado de São Paulo. Revista Brasileira de Fruticultura, Jaboticabal, v.24, n.3, p.611-614, 2002.

GEORGE, A.P.; NISSEN, R.J. Effects of growth regulants on defoliation, flowering, and fruit maturity of the low chill peach cultivar Flordaprince in subtropical Australia. Australian Journal of Experimental Agriculture, Collingwood, v.33, p.787-795, 1993. 
GOLDBACK, H.; THALER, C.; WÜNSCH, A. Decomposition of 14C- labeled cyanamide in Vitis vinifera cuttings. Journal of Plant Physiology, Elsevier, v.133, p.299-303, 1988.

MIELE, A. Efeito da cianamida hidrogenada na quebra de dormência das gemas, produtividade do vinhedo e composição química do mosto da uva Cabernet Sauvignon. Pesquisa Agropecuária Brasileira, Brasília, v.26, n.3, p.315-354, 1991.

OMRAN, R.G. Peroxide levels and the activities of catalase, peroxidase, and indoleacetic acid oxidase during and after chilling of cucumber seedings. Plant Physiology, Rockville, v.65, p.407408, 1980.

PETRI, J.L.; PALLADINI, L.A.; SCHUCK, E.; DUCROQUET, J.H.J.; MATOS, C.S.; POLA, A.C. Dormência e indução de brotação de fruteiras de clima temperado. Florianópolis: Epagri, 1996. $110 \mathrm{p}$.
PIRES, E.J.P. Emprego de Reguladores Vegetais de Crescimento em Viticultura Tropical. Informe Agropecuário, Belo Horizonte, v.19, n.194, p.40-43, 1998.

PIRES, E.J.P.; POMMER, C.V.; TERRA, M.M.; PASSOS, I.R.S Effects de la cyanamide de calcium et de la cyanamide hydrogéne sur la levée de dormance dês bourgeons, lê débourrement et lê rendement du cépage Niágara Rose dans la région de Jundiaí, État de São Paulo, Brésil. Bulletin de L' O.I.V., Paris, v.72, n.821822, p.457-483, 1999.

REDDY, N.N.; SHIKHAMANY, S.D. Effect of hydrogen cyanamide and thiourea on budbreak and bloom of Thompson Seedless grapevines under tropical conditions. Crop Research, Hisar, v.2, n.2, p.163-168, 1989.

SHULMAN, Y.; NIR, G.; LAVEE, S. Oxidative processes in bud dormancy and the use of hydrogen cyanamide in breaking dormancy. Acta Horticulturae, Belgium, v.179, p.141-148, 1986. 